\title{
Effects of Cognitive Nursing Combined with Continuous Nursing on Postpartum Mental State and Rehabilitation
}

\author{
Caijuan Liu, ${ }^{1}$ Dailing Xiao, ${ }^{1}$ Deping Han, ${ }^{1}$ Shimin Li $\mathbb{D}^{2}{ }^{2}$ Tianli Zhu, ${ }^{3}$ Wenjuan Wang, \\ Li Zhou, ${ }^{3}$ Wen Yan, ${ }^{3}$ and Weiming Lu $\mathbb{D i D}^{4}$ \\ ${ }^{1}$ Chengyang People's Hospital, Qingdao, China \\ ${ }^{2}$ Department of Operation room, Jinan Maternal \& Children Health Care Hospital, Jinan, Shangdong, China \\ ${ }^{3}$ Department of Obstetric ward 2, Xinhua Hospital Affiliated to Medical College of Shanghai Jiaotong University, \\ Shanghai 200092, China \\ ${ }^{4}$ Department of Obstetrics ward 1, Xinhua Hospital Affiliated to Medical College of Shanghai Jiaotong University, \\ Shanghai 200092, China
}

Correspondence should be addressed to Shimin Li; lishimin1232021@163.com and Weiming Lu; weiming_lu01@163.com

Received 27 May 2021; Revised 15 September 2021; Accepted 22 September 2021; Published 2 December 2021

Academic Editor: Dorota Formanowicz

Copyright (C) 2021 Caijuan Liu et al. This is an open access article distributed under the Creative Commons Attribution License, which permits unrestricted use, distribution, and reproduction in any medium, provided the original work is properly cited.

\begin{abstract}
Purpose. This study is aimed at exploring the effects of cognitive nursing combined with continuous nursing on postpartum mental state and rehabilitation. Methods. Totally, 124 puerperas admitted to our hospital from January 2019 to January 2020 were selected and divided into a research group and a control group according to different nursing methods, with 62 cases in each group. The control group received routine care, while the research group received cognitive nursing combined with continuous nursing on this basis. The mental state, rehabilitation indicators, quality of life, incidence of complications, and nursing satisfaction were compared between the two groups after intervention. Results. Before nursing, there was no statistically significant difference in the SAS and SDS scores between the two groups $(P>0.05)$; after intervention, the SAS and SDS scores of the two groups were significantly reduced, and those of the research group were lower than those of the control group $(P<0.05)$. After intervention, the time of the first breastfeeding, duration of lochia rubra, length of hospital stay, and score of uterine contraction pain of the research group were lower than those of the control group $(P<0.05)$; the psychological function, physical function, material life, and social function scores of the research group were higher than those of the control group $(P<0.05)$; the incidence of complications in the research group was $4.84 \%$, lower than $20.97 \%$ in the control group $(P<0.05)$; the nursing satisfaction of the research group was $96.77 \%$, which was significantly higher than $83.87 \%$ in the control group $(P<0.05)$. Conclusions. Cognitive nursing combined with continuous nursing can effectively improve the mental state, shorten the length of hospital stay, increase the perceived well-being, and promote the physical rehabilitation in puerperas, which is worth promoting in clinical practice.
\end{abstract}

\section{Introduction}

A puerpera refers to a woman in the puerperium and parturition period. This is a necessary stage in the life activities of most puerperas and is often accompanied by a series of obvious psychological and physical stress reactions [1]. Due to the difficulty in adapting to the mother's role, complex emotions, and excessive worries, some puerperas have psychological problems such as anxiety and depression. Coupled with abdominal weight and fetal movement, these problems often produce negative emotional experiences, elevate the heart rate and blood pressure of puerperas, lead to sleep disorders and other adverse events, increase the risks of uterine inertia and postpartum contractions, and compromise the delivery process and the postpartum recovery of puerperas [2,3]. Conventional obstetric nursing interventions in clinical practice often ignore the mental state and needs of puerperas and cannot ameliorate their negative state completely [4]. Therefore, we have been 
TABLE 1: SAS and SDS scores of the two groups before and after intervention $\left({ }^{-} \chi \pm s\right)$.

\begin{tabular}{lcccc}
\hline Group & \multicolumn{2}{c}{ SAS scores } & \multicolumn{2}{c}{ SDS scores } \\
& Before nursing & After intervention & Before nursing & After intervention \\
\hline Research group $(n=62)$ & $62.63 \pm 9.15$ & $38.44 \pm 6.85^{\mathrm{a}}$ & $61.58 \pm 9.22$ & $36.24 \pm 6.05^{\mathrm{a}}$ \\
Control group $(n=62)$ & $62.68 \pm 9.11$ & $44.68 \pm 8.07^{\mathrm{a}}$ & $61.62 \pm 9.30$ & $43.29 \pm 8.68^{\mathrm{a}}$ \\
$t$ & 0.030 & 4.642 & 0.024 & 5.247 \\
$P$ & 0.976 & $<0.001$ & 0.980 & $<0.001$ \\
\hline
\end{tabular}

Note: a represented that compared with that before nursing, $P<0.05$.

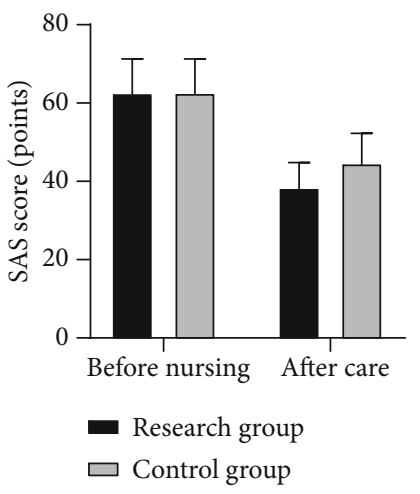

FIGURE 1: SAS scores of the two groups before and after intervention.

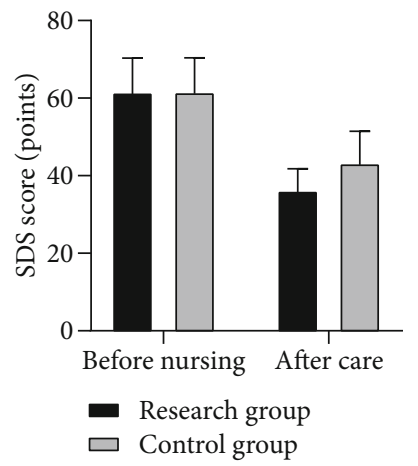

FIGURE 2: SDS scores of the two groups before and after intervention.

exploring a more effective postpartum nursing intervention for clinical practice. In recent years, as the living standards are improved, people have higher requirements for the quality of nursing services, and more humane, refined, and higherquality nursing interventions are needed for puerperas in clinical practice. Cognitive nursing enables patients to have correct cognitive behaviors by correcting wrong cognition and ultimately alleviates the condition and treats the disease [5]. Continuous nursing is the continuation of hospital care. It is a people-oriented, scientific, and systematic model that can provide professional guidance on infant care and health care for a certain time after discharge from the hospital and effectively improve the physical and mental health as well as the quality of life of puerperas [6]. There are few studies on cognitive nursing combined with continuous nursing. Hence, in this study, a total of 124 puerperas admitted to our hospital from January
2019 to January 2020 were selected to explore the effects of cognitive nursing combined with continuous nursing on postpartum mental state and rehabilitation of puerperas.

\section{Materials and Methods}

2.1. Subjects. Totally, 124 puerperas admitted to our hospital from January 2019 to January 2020 were selected as the subjects. The puerperas were divided into research group and control group, with 62 cases in each group. Randomisation was stratified by center with minimisation for age, gestational age, education level, and gravidity. The control group: 22-36 years old, with an average of $(29.73 \pm 4.28)$ years; the gestational age was $37-40$ weeks, with an average of $(39.25 \pm 1.52)$ weeks; 33 postpartum women and 29 primiparous women; 14 cases with junior high school education, 19 with senior high school education, and 29 with university education. The research group: 21-37 years old, with an average of $(29.80 \pm 4.30)$ years; the gestational age was $36-40$ weeks, with an average of $(39.05 \pm 1.43)$ weeks; 33 postpartum women and 29 primiparous women; 15 cases with junior high school education, 21 cases with senior high school education, and 26 cases with university education. There was no statistically significant difference in the general data between the two groups $(P>0.05)$. This study was approved by the appropriate ethics committee of our hospital.

Inclusion criteria: [1] puerperas who gave singleton birth naturally; [2] complete clinical data; [3] patients' informed consent. Exclusion criteria: [1] severe complications in childbirth; [2] severe heart, liver, kidney, and other organ diseases; [3] mental and cognitive-communication deficits.

2.2. Methods. The control group received routine nursing care as follows. The self-made postpartum care manual of our hospital was distributed and explained to the puerperas, and psychological counseling was provided to them. They and their families were informed of the hotline of the obstetrics department so that they could consult the department at any time. Their contact information was recorded for follow-up.

The research group underwent cognitive nursing combined with continuous nursing on the basis of the control group as follows. [1] A joint cognitive and continuous nursing team was established, with the head nurse as the team leader. Comprehensively understood the basic information of patients and families, communicate with patients, and analyze the psychological dynamics of patients. The root causes of patients with depression were evaluated. [2] 
TABLE 2: Comparison of the postpartum physiological state between the two groups $\left({ }^{-} \chi \pm s\right)$.

\begin{tabular}{lcccc}
\hline Group & $\begin{array}{c}\text { Time of the first } \\
\text { breastfeeding }\end{array}$ & $\begin{array}{c}\text { Duration of lochia rubra } \\
(\mathrm{h})\end{array}$ & $\begin{array}{c}\text { Length of hospital stay } \\
(\mathrm{h})\end{array}$ & $\begin{array}{c}\text { Score of uterine contraction } \\
\text { pain }\end{array}$ \\
\hline $\begin{array}{l}\text { Research group } \\
(n=62)\end{array}$ & $15.87 \pm 2.15$ & $2.00 \pm 1.01$ & $3.33 \pm 1.17$ & $2.90 \pm 1.30$ \\
Control group $(n=62$ & $23.63 \pm 2.84$ & $3.63 \pm 1.53$ & $6.05 \pm 1.25$ & $4.63 \pm 1.51$ \\
) & 17.150 & 7.001 & 12.510 & 6.837 \\
$t$ & $<0.001$ & $<0.001$ & $<0.001$ & $<0.001$ \\
$P$ & & & \\
\hline
\end{tabular}

TABLE 3: Comparison of the quality of life scores between the two groups after intervention $\left({ }^{-} \chi \pm s\right)$.

\begin{tabular}{lcccc}
\hline Group & Psychological function & Physical function & Material life & Social function \\
\hline Research group $(n=62)$ & $57.87 \pm 5.59$ & $64.82 \pm 6.33$ & $70.25 \pm 7.18$ & $67.22 \pm 6.16$ \\
Control group $(n=62)$ & $53.25 \pm 5.44$ & $59.36 \pm 6.06$ & $63.11 \pm 5.46$ & $60.36 \pm 5.77$ \\
$t$ & 4.664 & 4.906 & 6.233 & 6.400 \\
$P$ & $<0.001$ & $<0.001$ & $<0.001$ & $<0.001$ \\
\hline
\end{tabular}

TABle 4: Comparison of the incidence of complications between the two groups $(n, \%)$.

\begin{tabular}{lcccc}
\hline Group & Asitia & Insomnia & Mastitis & $\begin{array}{c}\text { Total } \\
\text { incidence }\end{array}$ \\
\hline $\begin{array}{l}\text { Research group } \\
(n=62)\end{array}$ & $\begin{array}{c}1 \\
(1.61)\end{array}$ & $2(3.23)$ & $0(0.00)$ & $3(4.84)$ \\
$\begin{array}{l}\text { Control group } \\
(n=62)\end{array}$ & 5 & $6(9.68)$ & $3(4.84)$ & $13(20.97)$ \\
$\chi^{2}$ & $(8.06)$ & & & 7.176 \\
$P$ & & & & 0.007 \\
\hline
\end{tabular}

Cognitive nursing care. The nurses communicated with the puerperas in time, encouraged them to tell their true emotions, listened to them carefully, analyzed their problems, corrected their distorted cognition, reshaped their cognition, and resolved their negative emotions such as depression and anxiety in an "empathetic" way. The family members were educated to increase their attention to the puerperas with care and encouragement to improve their self-confidence and cooperation. [3] A reasonable and scientific diet plan was developed according the health status of the puerperas, which included high-calorie, high-protein, high-vitamin, and easily digestible foods. [4] Continuous nursing care. A postdischarge nursing plan was formulated before the puerperas were discharged from the hospital, and a health record was established for them, for example, a return visit file to clarify the method, time, and content of return visits and record their relevant information. [5] The medical and nursing staff carried out a telephone follow-up within 1 week after the puerperas were discharged from the hospital to understand their current situation and provide health education and guidance on their life. The medical and nursing staff advised the family members to take care of the puerperas and take the initiative to take care of the newborns, so that the puerperas could face life with a positive attitude and avoid emotional fluctuations. [6] The medical and nursing staff could provide psychological counseling to the puerperas through text messages, phone calls, WeChat, etc., so that the puerperas could pour out their emotions. Meanwhile, the medical and nursing staff developed an exercise plan including respiratory training, postpartum yoga training for the research group to promote their recovery. [7] The medical and nursing staff conducted a home follow-up visit once a month to understand their family support and evaluate their mental and physical recovery and provided guidance for their future recovery.

\subsection{Observation Indicators}

2.3.1. Mental State. After one-month intervention, the mental state was evaluated. The Self-Anxiety Scale (SAS) and Self-Depression Scale (SDS) were used to evaluate the mental state of the puerperas. There were 40 items in SAS and SDS, each with an average of 20 items, and the score of each item was 1-4. SAS rating standards [7]: (1) $\geq 70$ scores $=$ severe anxiety; (2) $60-69$ scores = moderate anxiety; (3) 50 -59 scores = mild anxiety; and (4) <50 scores = normal. The higher the score, the more serious the anxiety. SDS rating standards [8]: (1) <50 scores = normal; (2) $50-59=$ mild depression; (3) 60-69= moderate depression; and (4) $\geq 70=$ severe depression. The higher the score, the more severe the depression.

2.3.2. Postpartum Rehabilitation Indicators. Postpartum rehabilitation indicators including the time of the first breastfeeding, duration of lochia rubra, length of hospital stay, and score of uterine contraction pain were collected and compared. Among them, the uterine contraction pain score was measured by the McGill Pain Questionnaire (MPQ). The MPQ score was 0-5: $0=$ no uterine contraction pain, $5=$ severe uterine contraction pain, and the degree of pain was proportional to the score. 
TABLE 5: Comparison of the satisfaction rate between the two groups $(n, \%)$.

\begin{tabular}{|c|c|c|c|c|}
\hline Group & Satisfactory & Basically satisfactory & Unsatisfactory & Satisfaction rate \\
\hline Research group $(n=62)$ & $48(77.42)$ & $12(19.35)$ & $2(3.23)$ & $60(96.77)$ \\
\hline Control group $(n=62)$ & $24(38.71)$ & $28(45.16)$ & $10(16.13)$ & $52(83.87)$ \\
\hline$\chi^{2}$ & & & & 5.905 \\
\hline$P$ & & & & 0.015 \\
\hline
\end{tabular}

2.3.3. Quality of Life. After one-month intervention, the quality of life was evaluated by the Generic Quality of Life Inventory-74 (GQOLI-74) [9], mainly divided into mental function, physical function, material life, and social function. The total score of GQOLI-74 was 100. The higher the score, the better the quality of life.

2.3.4. Complications. After one-month intervention, the complications including asitia, insomnia, and mastitis were monitored. The complication rate was obtained. Comparison of the incidence of complications between the two groups after intervention.

2.3.5. Nursing Satisfaction. The self-made satisfaction inventory of our hospital was used to evaluate the service attitude, communication skills, nursing skills, humane care, and health anonymously. The total score was 100 . A score of $>90$ was considered satisfactory; a score of 70-90 was basically satisfactory; a score of $<70$ was unsatisfactory. The higher the score, the higher the satisfaction. Satisfaction $=($ satisfaction rate + relatively satisfactory rate)/total number of cases $\times 100 \%$.

\section{Results}

3.1. Comparison of the Mental State of the Two Groups before and after Intervention. Before nursing, there was no statistically significant difference in SAS and SDS scores between the two groups $(P>0.05)$. After intervention, the SAS and SDS scores of the two groups were significantly reduced, and those of the research group were lower than those of the control group $(P<0.05)$; see Table 1 , Figures 1 and 2.

\subsection{Comparison of Postpartum Rehabilitation Indicators} between the Two Groups. After intervention, the time of the first breastfeeding, duration of lochia rubra, length of hospital stay, and score of uterine contraction pain of the research group were lower than those of the control group $(P<0.05)$; see Table 2 .

3.3. Comparison of the Quality of Life Scores between the Two Groups after Intervention. After intervention, the psychological function, physical function, material life, and social function scores of the research group were higher than those of the control group $(P<0.05)$; see Table 3 .

3.4. Comparison of the Incidence of Complications between the Two Groups after Intervention. The incidence of complications of the research group was $4.84 \%$, lower than $20.97 \%$ of the control group $(P<0.05)$; see Table 4 .
3.5. Comparison of Nursing Satisfaction between the Two Groups of Puerperas. The nursing satisfaction rate of the research group was $96.77 \%$, which was significantly higher than $83.87 \%$ of the control group $(P<0.05)$; see Table 5 .

\section{Discussion}

During pregnancy, most women are prone to life stress, hormone secretion, physical discomfort and excessive worries, and negative emotions such as depression and anxiety after delivery, thus, compromising the postpartum recovery of the puerperas and the relationship between them and their family [10]. According to research reports [11, 12], 3.5\% to $33 \%$ of puerperas in other countries and $4.5 \%$ to $20 \%$ in China have postpartum depression, which seriously threatens the health and safety of puerperas and babies. Due to lack of labor experience and knowledge, primiparas often have various worries during pregnancy, and due to the change of roles after delivery, some puerperas experience obvious loneliness [13]. Therefore, it is necessary to carry out nursing interventions for puerperas to relieve their negative emotions and promote their postpartum psychological and physical recovery.

In recent years, with the transformation of the nursing model, the concept of nursing care has undergone a major change from "disease-centered" to "people-centered," which gives play to the central role of puerperas in postpartum rehabilitation [14]. It scientifically extends the nursing service for puerperas from the hospital to the community and the family and does not affect the coordination and continuity between the nursing inside and outside the hospital, ensuring the continuity of nursing services for puerperas after leaving the hospital [15]. Continuous services are provided to puerperas after discharge, mainly by phone calls, text messages, and follow-ups. Health education on the healthcare knowledge of themselves and their newborns and psychological interventions are provided to puerperas, so as to make them aware of the harm of their own emotional changes and inform them of postpartum self-care and newborn care in time to reduce postpartum infections [16]. In addition, from the perspective of psychological defense mechanism, the doubts of the puerperas can be resolved by telephone at any time, which improves the adaptability during the puerperium, enhances the cognition of the puerperium healthcare and the nursing of the newborn, and prevents the occurrence of bad emotions caused by various factors. Meanwhile, text messages, WeChat, phone calls, and follow-up provide a window for the puerperas to release 
their psychological pressure and reduce their psychological burden. Relevant clinical studies have shown [17] that effective continuous nursing care for the puerperas after delivery can increase their knowledge of pelvic floor dysfunction, thereby arousing their enthusiasm and initiative. Cognitive nursing is a more effective treatment method, which can effectively alleviate negative mental states and reduce anxiety and depression and other negative behaviors and cognition [18]. Relevant articles have shown [6] that cognitive behavior can correct the negative thinking of puerperas, replace negative avoidance, change negative cognition, and effectively ameliorate anxiety and depression and other adverse symptoms. Hinkle et al. [19] has found that negative emotions during the puerperium may enhance the body's stress ability, and effective nursing interventions can reduce the negative emotions after delivery and the postpartum complications, and improve the mental state of puerperas. This study mainly explores the effect of cognitive nursing combined with continuous nursing on the postpartum mental state and rehabilitation of puerperas, and the nursing effect is definite. The results of this study showed that after intervention, the SAS and SDS scores of the two groups were significantly reduced, and those of the research group were lower than those of the control group, indicating that cognitive nursing combined with continuous nursing can significantly relieve postpartum depression. After intervention, the time of the first breastfeeding, duration of lochia rubra, length of hospital stay, and score of uterine contraction pain of the research group were lower than those of the control group, indicating that cognitive nursing combined with continuous nursing can speed up the physical recovery of puerperas. After intervention, the scores of psychological function, physical function, material life, and social function of the research group were higher than those of the control group, showing that cognitive nursing combined with continuous nursing can effectively improve the quality of life of puerperas. The complication rate of the research group was $4.84 \%$, lower than $20.97 \%$ of the control group, and the nursing satisfaction rate of the research group was $96.77 \%$, significantly higher than $83.87 \%$ of the control group, indicating that this nursing model can not only reduce the incidence of postpartum complications but also improve patient satisfaction.

In conclusion, cognitive nursing combined with continuous nursing can effectively improve the mental state of puerperas, shorten the length of hospital stay, enhance the health awareness, and promote the physical rehabilitation. It is worth promoting in clinical practice.

\section{Data Availability}

The data used to support the findings of this study are available from the corresponding author upon request.

\section{Conflicts of Interest}

The authors declare no conflict of interest.

\section{References}

[1] I. Rönnqvist, O. Brus, Å. Hammar et al., "Rehospitalization of postpartum depression and psychosis after electroconvulsive therapy: a population-based study with a matched control group," Journal of ECT, vol. 35, no. 4, pp. 264-271, 2019.

[2] B. Bodner-Adler, O. Kimberger, J. Griebaum, P. Husslein, and K. J. B. P. Bodner, "A ten-year study of midwife-led care at an Austrian tertiary care center: a retrospective analysis with special consideration of perineal trauma," BMC pregnancy and childbirth, vol. 17, no. 1, p. 357, 2017.

[3] C. Sunnqvist, K. Sjöström, and H. Finnbogadóttir, "Depressive symptoms during pregnancy and postpartum in women and use of antidepressant treatment-a longitudinal cohort study," International journal of women's health, vol. 11, pp. 109-117, 2019.

[4] K. Peltzer, J. M. Abbamonte, L. N. Mandell et al., "The effect of male involvement and a prevention of mother-to-child transmission (PMTCT) intervention on depressive symptoms in perinatal HIV-infected rural South African women," Archives of women's mental health, vol. 23, no. 1, pp. 101-111, 2020.

[5] E. C. Garman, A. Cois, M. Tomlinson, M. J. Rotheram-Borus, and C. Lund, "Course of perinatal depressive symptoms among South African women: associations with child outcomes at 18 and 36 months," Social psychiatry and psychiatric epidemiology, vol. 54, no. 9, pp. 1111-1123, 2019.

[6] H. Y. Kim, S. Y. Lee, S. Y. Choi, T. H. Kim, and H. J. Kim, “399: Prediction of adverse outcome of postpartum hemorrhage with shock index," Journal of Obstetrics \& Gynecology, vol. 218, no. 1, p. S244, 2018.

[7] L. Osborne, M. Clive, M. Kimmel et al., "Replication of epigenetic postpartum depression biomarkers and variation with hormone levels," Neuropsychopharmacology, vol. 41, no. 6, pp. 1648-1658, 2016.

[8] L. Kashani, S. Eslatmanesh, N. Saedi et al., "Comparison of saffron versus fluoxetine in treatment of mild to moderate postpartum depression: a double-blind, randomized clinical trial," Pharmacopsychiatry, vol. 50, no. 2, pp. 64-68, 2017.

[9] J. Julvez, M. Guxens, A. E. Carsin et al., "A cohort study on full breastfeeding and child neuropsychological development: the role of maternal social, psychological, and nutritional factors," Developmental Medicine \& Child Neurology, vol. 56, no. 2, pp. 148-156, 2014.

[10] L. M. Yee, N. G. Martinez, A. T. Nguyen, N. Hajjar, M. J. Chen, and M. A. Simon, "Using a patient navigator to improve postpartum care in an urban women's health clinic," Obstetrics and gynecology, vol. 129, no. 5, pp. 925-933, 2017.

[11] P. Thomas, "Collaborating sites for community-oriented integrated care and health promotion," London journal of primary care, vol. 9, no. 1, pp. 2-6, 2017.

[12] E. Mori, M. Tsuchiya, K. Maehara, H. Iwata, A. Sakajo, and K. Tamakoshi, "Fatigue, depression, maternal confidence, and maternal satisfaction during the first month postpartum: a comparison of Japanese mothers by age and parity," International journal of nursing practice, vol. 23, no. 1, article e12508, 2017.

[13] E. Hoekzema, E. Barba-Müller, C. Pozzobon et al., "Pregnancy leads to long-lasting changes in human brain structure," Nature neuroscience, vol. 20, no. 2, pp. 287-296, 2017.

[14] A. Małus, J. Szyluk, B. Galińska-Skok, and B. Konarzewska, "Incidence of postpartum depression and couple relationship quality,” Psychiatr Pol, vol. 50, no. 6, pp. 1135-1146, 2016. 
[15] J. M. Hartmann, R. A. Mendoza-Sassi, and J. A. Cesar, "Depressão entre puérperas: prevalência e fatores associados," Cadernos de saude publica, vol. 33, no. 9, 2017.

[16] H. Youn, S. Lee, S. W. Han et al., "Obstetric risk factors for depression during the postpartum period in South Korea: a nationwide study," Journal of Psychosomatic Research, vol. 102, pp. 15-20, 2017.

[17] M. Thomson and V. Sharma, "Therapeutics of postpartum depression," Expert review of neurotherapeutics, vol. 17, no. 5, pp. 495-507, 2017.

[18] I. Mohanty and T. A. Gebremedhin, "Maternal autonomy and birth registration in India: who gets counted?," PloS one, vol. 13, no. 3, article e0194095, 2018.

[19] S. N. Hinkle, G. M. Buck Louis, S. Rawal, Y. Zhu, P. S. Albert, and C. Zhang, "A longitudinal study of depression and gestational diabetes in pregnancy and the postpartum period," Diabetologia, vol. 59, no. 12, pp. 2594-2602, 2016. 